\section{Comparison of Radiation Resistant among Polysulfones Prepared from Several Aromatic Diol and 4,4'-Dichlorodiphenyl Sulfone}

Hideharu Shintani*

Faculty of Science and Engineering, Chuo University, Tokyo, Japan

\begin{abstract}
An aromatic polysulfone consists of 4,4'-diolaromatic compound and 4,4'-dichlorodiphenyl sulfone. As 4,4'diol aromatic compounds, there exists bisphenol A, p-dihydroxy benzene, 4,4'-diphenol methane and p,p'-diphenol (bisphenol) and they were compared to study which compound would indicate the most resistant to gamma-ray exposure. Sulfur dioxide $\left(\mathrm{SO}_{2}\right)$ from 4,4'-dichlorodiphenyl sulfone was used as an indicator for evaluation. The use of bisphenol in the preparation of polysulfone indicated the most resistant to the gamma-ray irradiation exposure and least production of $\mathrm{SO}_{2}$ among aromatic diol compounds tested. This indicated polysulfone free from bisphenol A is producible. The production of $\mathrm{SO}_{2}$ from bisphenol-based polysulfone was around $43 \%$ of bisphenol $\mathrm{A}$ based polysulfone. The rate of decrease of tensile strength correlated well with the order of radiation resistance. The fracture toughness of bisphenol A based polysulfone decreased with irradiation dose, but the bisphenol based polysulfone maintained its original ductility.
\end{abstract}

Keywords: Radiation; Sterilization; Polysulfone; Bisphenol A

\section{Introduction}

Bisphenol A is known as an endocrine disrupter. However, this compound is now mostly used for production of polysulfone and polycarbonate in the field of medical device preparation. When bisphenol A based polysulfone or bisphenol A based polycarbonate was sterilized with gamma-ray irradiation, autoclaving at $121.1^{\circ} \mathrm{C}$ for more than $20 \mathrm{~min}$, or ozone gas exposure, bisphenol A was produced after cleavage of polysulfone or polycarbonate $[1,2]$. This phenomenon is not desirable. This cleavage must be protected to be safe for humans from the exposure of hazardous compound and free from environmental deterioration. There is an increasing need and demand for medical polymers free from hazardous chemicals and medical polymers to be tolerant for several sorts of sterilization procedures for production of medical devices. Biosafety and biocompatible characteristics are additionally required for safety healthcare products production. There have been relatively few systematic studies of the relationship between polymer structure and radiation resistance for polymers with aromatic backbones. Such investigations are necessary to determine which linkages are the most susceptible to degradation by radiation exposure. All of the polymers tested are newly synthesized and are linear with no long chain branching. All the polymers tested have similar molecular weights and molecular weight distributions, which allow the comparison of the change in mechanical properties with radiation and polymer structure to be a more defined structure/ property investigation.

The mechanical properties exhibited by a polymer after irradiations are a complex function. Therefore, it is hard to draw structure/radiation resistance conclusions only from the change in the mechanical properties. However, the changes in mechanical properties are direct indications of the ultimate usefulness of the polymer in a radiation environment. In this paper, the authors examined the relationship between radiation resistance and polymer structure using volatile product determination and mechanical property measurements.

\section{Materials and Methods}

The polymers were synthesized by a nucleophilic activated aromatic substitution reaction of an aromatic diol compound and $4,4^{\prime}$-dichloro diphenylsulfone [2,3]. The reaction was carried out in a dipolar solvent ( $\mathrm{N}$-methylpyrrolidone, NMP) at $170^{\circ} \mathrm{C}$ in the presence of potassium carbonate (Figure 1) [3,4]. The polymers were purified by repeated precipitation into methanol/water, followed by drying. The aromatic diol compounds used were bisphenol A, p-dihydroxy benzene, 4,4'-diphenol methane and p,p'-diphenol (bisphenol). The composition and structures of these polymers were characterized by $1 \mathrm{H}$ and 13C NMR (nuclear magnetic resonance) and infrared spectroscopy (IR) to confirm that linear, all p (para) substituted polymers were confirmed. The glass transition temperature was determined on a DSC-2 (Differential Scanning Calorimetry from Perkin-Elmer) using a heating rate of $10 \mathrm{oC} / \mathrm{min}$ and the intrinsic viscosity was determined in NMP (N-methylpyrrolidone) at $25^{\circ} \mathrm{C}$.

The technique used for volatile product analysis was based on the quantitative transfer of volatile small molecule compounds, which were produced by radiolysis of the polymer onto a GLC column where they are separated, identified and determined quantitatively. A weighed sample of $40 \mathrm{mg}$ of polymer was irradiated and put into the ampoule. The ampoule was heated to apply for headspace GLC (gas liquid chromatography) analysis using Perkin Elmer HS-40. The gases to be tested were injected onto a carrier medium of Chromosorb 102 column together with helium carrier gas and FID (flame ionization detector). $6^{\circ} \mathrm{C}$ gamma irradiation was conducted with dose rates of approximately $10 \mathrm{kGyper} h$.

\section{Results and Discussion}

The major volatile product from the irradiation of bisphenol A based polysulfone at $150^{\circ} \mathrm{C}$ was sulfur dioxide $\left(\mathrm{SO}_{2}\right)$ which was produced with $\mathrm{G}(\mathrm{SO})=0.146 .22$. The $\mathrm{G}$ value indicates the amount of produced product when $100 \mathrm{eV}$ energy was absorbed to the polymer. Other volatile products observed were hydrogen, methane, and carbon dioxide. The $\mathrm{G}$ value of $\mathrm{SO}_{2}$ was much greater and more selective than that of other gases $\left(\mathrm{H}_{2}, \mathrm{CH}_{4}\right.$ or $\left.\mathrm{CO}_{2}\right)$ produced upon irradiation. This

${ }^{*}$ Corresponding author: Hideharu Shintani, Faculty of Science and Engineering Chuo University, 1-13-27, Kasuga, Bunkyo, 112-8551, Tokyo, Japan, Tel: +81425922336, Fax: +81425922336; E-mail: shintani@mail.hinocatv.ne.jp

Received June 07, 2014; Accepted June 23, 2014; Published July 02, 2014

Citation: Shintani H (2014) Comparison of Radiation Resistant among Polysulfones Prepared from Several Aromatic Diol and 4,4'-Dichlorodiphenyl Sulfone. Pharmaceut Reg Affairs 3: 124. doi:10.4172/2167-7689.1000124

Copyright: ( 2014 Shintani H. This is an open-access article distributed under the terms of the Creative Commons Attribution License, which permits unrestricted use, distribution, and reproduction in any medium, provided the original author and source are credited. 


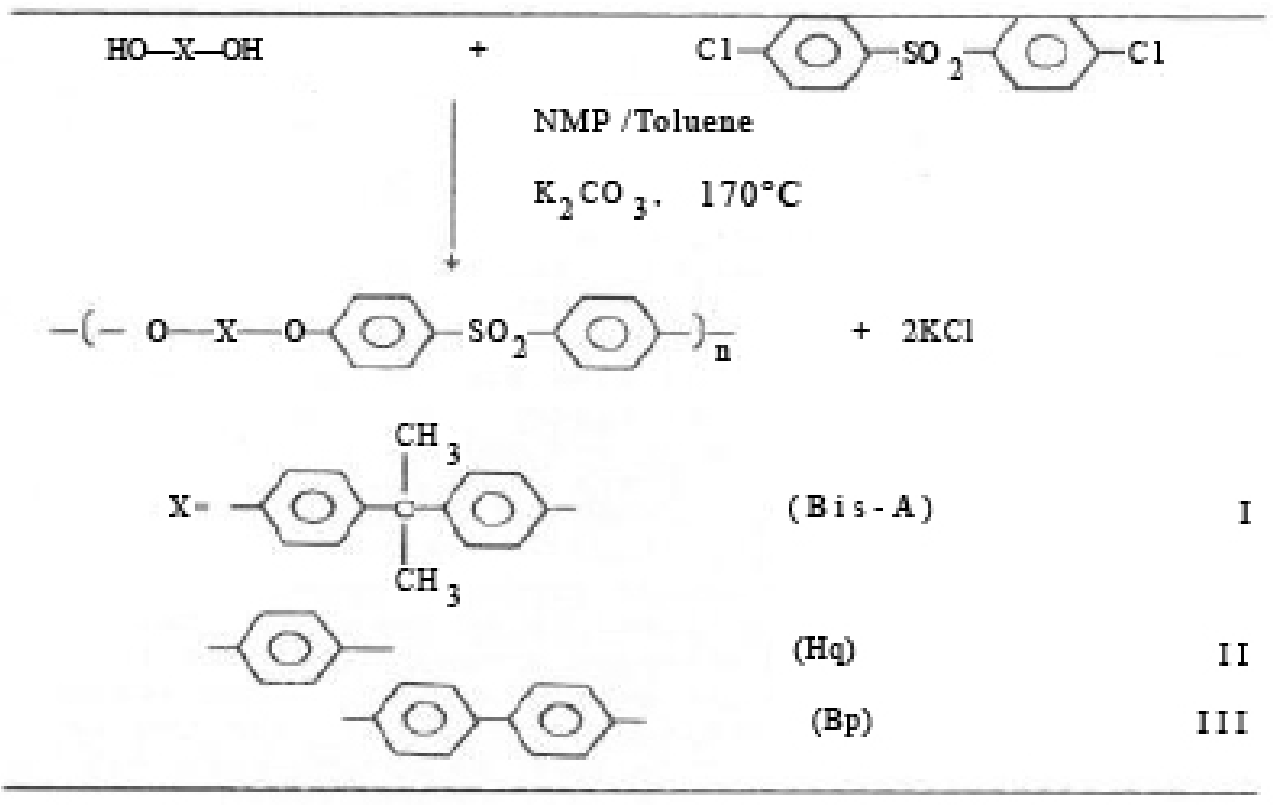

Figure 1: Preparation procedure of polysulfone using several aromatic diol compounds and 4,4'-dichlorodiphenyl sulfone.

is the reason $\mathrm{G}\left(\mathrm{SO}_{2}\right)$ was defined as an indicator of the aromatic diol compound based polysulfone degradation upon irradiation.

Elimination of a methyl radical from the isopropylidene group of bisphenol A based polysulfone, followed by hydrogen abstraction to form methane was observed but not abundant. This is because the isopropylidene group is a relatively radiation resistant functional group in a chemical structure of bisphenol A. Therefore, even though small amount of methane $\left(\mathrm{CH}_{4}\right)$ production from irradiated bisphenol A based polysulfone was recognized, but the $\mathrm{G}\left(\mathrm{CH}_{4}\right)$ was very small compared with $\mathrm{G}\left(\mathrm{SO}_{2}\right)$. This indicates that $\mathrm{C}-\mathrm{CH}_{3}$ scission in isopropylidene group of bisphenol A is relatively lower. Methyl group in isopropylidene group of bisphenol A based polysulfone serves for transparency of aromatic diol compound based polysulfone. The dependence of the volatile product yield with structure can be a very sensitive probe of radiation resistance and the protective effect of aromatic rings. Since all polymers examined contained the sulfone moiety and $\mathrm{SO}_{2}$ production indicated greatest amount among the gases produced. The $\mathrm{G}\left(\mathrm{SO}_{2}\right)$ comparison data is presented in (Table 1).

The stability of the isopropylidene group upon the radiation degradation of bisphenol A based polysulfone resulted in the small difference in $\mathrm{G}\left(\mathrm{SO}_{2}\right)$ for bisphenol A based polysulfone $(\mathrm{G}=0.146)$ and p-diphdroxybenzene based PS $(\mathrm{G}=0.136)$ (Table 1$)$. The relatively small reduction in $\mathrm{G}\left(\mathrm{SO}_{2}\right)$ for the latter may be due to a slightly higher aromatic content compared with the former. The presence or absence of isopropylidene functional group is not a major factor in this case.

The most radiation resistant polysulfone investigated was bisphenol based polysulfone, $\mathrm{G}\left(\mathrm{SO}_{2}\right)$ of 0.063 . Although the sulfone group is not directly attached to the bisphenol (Figure l), there is evidently a large protective effect either through space or along the polymer chain. As the total aromatic group content of bisphenol based polysulfone was only slightly higher than for bisphenol A based polysulfone and the indicator of the amount of aromatic functional group content in polysulfone did not significantly contribute to the radiation resistance.

The presence or absence of isopropylidene functional group as well as the aromatic group content in polysulfone is not an appropriate indicator to compare. The considered possibility is the radical distribution. The radical cations and anions formed immediately after irradiation are stabilized to a greater extent in the bisphenol case than for a single dihydroxy benzene ring. The aromatic rings in the bisphenol are nearly $90^{\circ}$ out of the plane of the molecule, which suggests that there is little interaction between these rings. After ionization with gamma ray or electron beam, the orientation of the rings could be altered, which would allow greater interaction and thus charge delocalization. This proposed reorientation might be the major factor for the enhanced stability. $\mathrm{G}\left(\mathrm{SO}_{2}\right)$ for p,p'-diphenolmethane based polysulfone was intermediate. This phenomenon could also be explained from the degree of charge delocalization after gamma-ray irradiation. Compared with bisphenol, methylene in $\mathrm{p}$, $\mathrm{p}^{\prime}$-diphenolmethane will inhibit charge delocalization. This may result in the fact that $\mathrm{G}\left(\mathrm{SO}_{2}\right)$ of p,p'-diphenolmethane was greater than that of bisphenol based polysulfone. In addition, methyl in isopropylidene indicated the steric inhibition of ion charge delocalization, thus $\mathrm{G}\left(\mathrm{SO}_{2}\right)$ of bisphenol A based polysulfone was greater than that of bisphenol based polysulfone or $\mathrm{p}, \mathrm{p}^{\prime}$-diphenolmethane based polysulfone. This indicated in place of bisphenol A based polysulfone, bisphenol based polysulfone or p,p'-diphenolmethane based polysulfone can be attainable and more desirable.

Following this procedure, polysulfone free from bisphenol A can be prepared and these prepared polysulfones are safer and more tolerable to gamma-ray irradiation exposure. Ultimately, it is the retention of mechanical properties after irradiation, which will determine the suitability of a polymer for use in a radiation environment. Since the potential applications for this class of polymer require high modulus and toughness over an extended dose range. One physical indicator is Young's modulus (Table 2). Young's modulus increased significantly after a dose of $500 \mathrm{kGy}$, but increased only slightly more at higher doses. The increase is attributed to the radiation induced network formation [4,5] (Table 2). The modulus for p,p'-diphenolmethane based polysulfone increased in a similar manner, suggesting similarity in the crosslinking reactions of these two polymers of bisphenol based polysulfone and p,p'-diphenolmethane based polysulfone. The 


\begin{tabular}{|c|c|}
\hline Polymer & $\mathrm{G}\left(\mathrm{SO}_{2}\right)$ \\
\hline bisphenol A-PS & $0.146 \pm 0.014$ \\
\hline p-dihydroxybenzene-PS & $0.136 \pm 0.011$ \\
\hline p,p'-dihydroxybenzene-PS & $0.097 \pm 0.004$ \\
\hline p,p'-diphenol-PS & $0.063 \pm 0.010$ \\
\hline
\end{tabular}

PS indicates polysulfone

Table 1: $\mathrm{G}\left(\mathrm{SO}_{2}\right)$ for aromatic diol compounds based polysulfone after gamma-ray Irradiation.

\begin{tabular}{|c|c|c|c|c|}
\hline Dose (kGy) & \multicolumn{2}{|c|}{ Bisphenol A-PS } & \multicolumn{2}{c|}{ p,p'-diphenylmethane-PS } \\
\hline & Modulus (MPa) & Elongation (\%) & Modulus (Mpa) & Elongation (\%) \\
\hline 0 & 1200 & 110 & 1163 & 74 \\
\hline 500 & 1660 & 80 & 1650 & 100 \\
\hline 1000 & 1750 & 33 & 1650 & 70 \\
\hline 2000 & 1600 & 20 & 1750 & 60 \\
\hline 4000 & 1880 & 8 & 1700 & 50 \\
\hline
\end{tabular}

Table 2: Changes of mechanical factors with irradiation doses.

mechanical property, which is most sensitive to radiation degradation, is the elongation at failure. This invariably decreases regardless of whether chain scission or crosslinking is predominant. For bisphenol A based polysulfone, the elongation at failure decreases rapidly from $110 \%$ initially to $8 \%$ after a dose of $4000 \mathrm{kGy}$ at ambient temperature (Table 2). The production of bisphenol A is increased with increasing radiation doses [1].

For p,p'-diphenolmethane based polysulfone, the decrease in the elongation at failure with irradiation dose is less than for bisphenol A based polysulfone. This phenomenon can be explained from the difference between isopropylidene functional group and methylene group based on steric inhibition, as demonstrated from $\mathrm{G}\left(\mathrm{SO}_{2}\right)$ difference, which depend on charge delocalization (Table 1). The decrease in the elongation at failure suggested that the fracture toughness might also decrease after irradiation. This was confirmed from the Fracture toughness (KIC) measurements, which showed a decrease from an initial value of $2.0 \times 106 \mathrm{Nm}-1.5$ to $1.7 \times 106 \mathrm{~N}$ m-1.5 after $1000 \mathrm{kGy}$. The decrease in KIC correlated with the decrease in tensile elongation at failure. A final value of 1,4 X106 Nm-1.5 after $4000 \mathrm{kGy}$ was observed. Fracture toughness may correlate with the $\beta$ relaxation temperature for the polymer. After irradiation, the $\beta$ relaxation temperature increased with a corresponding broadening and decrease in intensity [6].

\section{Conclusion}

This research demonstrates the utility of a well-defined set of polymers with carefully controlled structure for relating structure to radiation resistance. The presence of the isopropylidene group in the polymer apparently had little effect on the radiation resistance of the polymer, as determined from volatile product yields. Methane production from the isopropylidene group was extremely small, indicating that isopropylidene bond scission is of a low probability and bisphenol A functional group was stable to gamma-ray irradiation. This was furthermore confirmed from
$\mathrm{G}\left(\mathrm{SO}_{2}\right)$ measurements. Bisphenol based polysulfone was the most radiation resistant polysulfone studied. This attributed to the least delocalization of the radical cations and anions formed immediately after absorption of energy by the bisphenol group. The protective effect of this group apparently acts either intramolecularly or through space, since the principal radiation induced volatile product, sulfur dioxide, $\mathrm{SO}_{2}$, is derived from the sulfone group, which is not adjacent to the bisphenol group (Figure 1). The overall aromatic content of bisphenol-based polysulfone is similar to bisphenol A based polysulfone and this difference is not clearly enough to explain the enhanced radiation resistance of bisphenol based polysulfone observed. These indicate degree of ion delocalization after irradiation is a major factor for evaluation. In place of bisphenol A based polysulfone, bisphenol based or p,p'-diphenolmethane based polysulfone can be applicable for polysulfone production. By using the latter two polysulfones, hazardous bisphenol A can be omitted from polysulfone production. These two polysulfones are biosafety polymers even though transparency of these two polysulfones may be somewhat inferior to the bisphenol. A based polysulfone. The increase in the modulus for bisphenol based polysulfone and p,p'diphenylmethane based polysulfone with irradiation indicated that crosslinking predominated for both polymers. For both polymers of bisphenol A based polysulfone and p,p'-diphenylmethane based polysulfone, there was an initial rapid decrease in the elongation at failure followed by a slower decrease. This effect was also demonstrated by the variation in the fracture toughness (KIC) with irradiation.

\section{References}

1. Shintani H (2001) Determination of the endocrine disrupter bisphenol-A in the blood of uremia patients treated by dialysis, Chromatographia 53: 331-333.

2. Shintani H, Suzuki E, Sakurai M (2003) Determination of Compounds Inhibiting Bacterial Growth in Sterilized Medical Devices, Chromatographia 58: 193-199.

3. Viswanathan R, Johnson BC, McGrath JE (1984) Synthesis, kinetic observations and characteristics of polyarylene ether sulphones prepared via a potassium carbonate DMAC process, Polymer 25: 1827-1831.

4. Hedrick JL, Mohanty DK, Johnson BC, Viswanathan R, Hinkley JA, et al. (1986) Radiation resistant amorphous-all aromatic polyarylene ether sulfones: Synthesis, characterization, and mechanical properties, J Polym Sci Chem 23: 287-300.

5. Sasuga T, Hayakawa N, Yoshida K, Hagiwara M (1985) Degradation in tensile properties of aromatic polymers by electron beam irradiation, Polymer 26 : 1039-1042.

6. Hinkley JA, J Polym Sci Polym Lett Ed 22: 497-500. 